\title{
The Effects of Channel Errors on Coordinated and Non-Coordinated Medium Access Control Protocols
}

\author{
Tolga Numanoglu, Student Member, IEEE, Bulent Tavli, Student Member, IEEE \\ Wendi Heinzelman, Member, IEEE
}

\begin{abstract}
In this paper, we investigate the effects of channel noise on the performance of coordinated and non-coordinated MAC protocols. Comparative evaluations of these protocols under a perfect channel assumption have shown that coordinated MAC protocols, which regulate channel access locally, outperform noncoordinated channel access schemes in terms of energy efficiency and throughput. However, coordinated MAC protocols are more vulnerable than non-coordinated MAC protocols to channel noise due to their dependence on the robustness of the control traffic. In order to observe the degradation in performance of a coordinated MAC protocol (MH-TRACE), we investigate the impact of losing control packets. Furthermore, the performance in terms of throughput, delay, and energy efficiency of both coordinated (MH-TRACE) and non-coordinated (IEEE 802.11) MAC protocols is explored using a general error model that takes into account the length of the packets. Our results show that despite its higher level of vulnerability, the coordinated MAC protocol's performance is superior to the performance of the non-coordinated MAC protocol even when error rates are high.
\end{abstract}

Index Terms-Energy-aware systems, distributed networks, wireless communication, network protocols, protocol verification, algorithm/protocol design and analysis, mobile communication systems, access schemes.

\section{INTRODUCTION}

In wireless communications, the channel, which is the common interface that connects the nodes, is a shared resource. Thus, access to this shared resource needs to be regulated; this resource allocation operation is performed by Medium Access Control (MAC) protocols, which are defined as the second layer of the OSI protocol stack [1]. The objective of controlling access to the channel via the MAC protocol is to avoid or minimize simultaneous transmission attempts (that will result in collisions) while maintaining a stable and efficient operating region for the whole network. Furthermore, the MAC protocol is the key element in determining many features of a wireless network, such as throughput, Quality of Service (QoS), energy dissipation, fairness, stability, and robustness [2].

MAC protocols can be classified into two categories based on the collaboration level of the network in regulating the channel access: coordinated and non-coordinated. A coordinated MAC protocol operates with explicit coordination among the nodes and is generally associated with coordinators, channel access schedules and clusters. For example, Bluetooth is a coordinated MAC protocol, where channel access within a cluster (i.e., piconet) is coordinated by a coordinator (i.e.,

Manuscript received January 6, 2005. This work was supported in part by the University of Rochester Center for Electronic Imaging Systems and in part by Harris Corporation, RF Communications Division.

The authors are with the Department of Electrical and Computer Engineering, University of Rochester, Rochester, NY 14627 USA (phone: 585-2758078, fax: 585-273-4919, e-mail: numanog1@ece.rochester.edu). piconet Master) [3]. A non-coordinated MAC protocol, on the other hand, operates without any explicit coordination among the nodes in the network. For example, IEEE 802.11 is a noncoordinated MAC protocol when operating in the broadcast mode (i.e., in broadcasting mode, IEEE 802.11 becomes plain CSMA without any handshaking) [4]. Note that IEEE 802.11 channel access in unicasting mode is a coordinated scheme (i.e., the four way handshaking between the transmitter and receiver is a special case of a general explicit coordination scheme, such as [5], [6]).

Figure 1 illustrates the channel access mechanism for generic coordinated and non-coordinated MAC protocols. In the coordinated MAC protocol, node $\mathrm{N}_{0}$ is the clusterhead (coordinator) for the portion of the network consisting of five nodes. Channel access is regulated through a schedule that is broadcast by the coordinator. Upon reception of the schedule, nodes transmit their data at their allocated time, and thus collisions among nodes within the same cluster are eliminated. Time is organized into cyclic time frames, and the transmission schedule is dynamically updated at the beginning of each time frame. IEEE 802.15.3 is a recent example of such a coordinated MAC protocol [7]. In the non-coordinated MAC protocol, each node determines its own transmission time based on feedback obtained through carrier sensing on the channel. Thus, conflicts in data transmission attempts (i.e., collisions, capture) are unavoidable in the non-coordinated scheme.

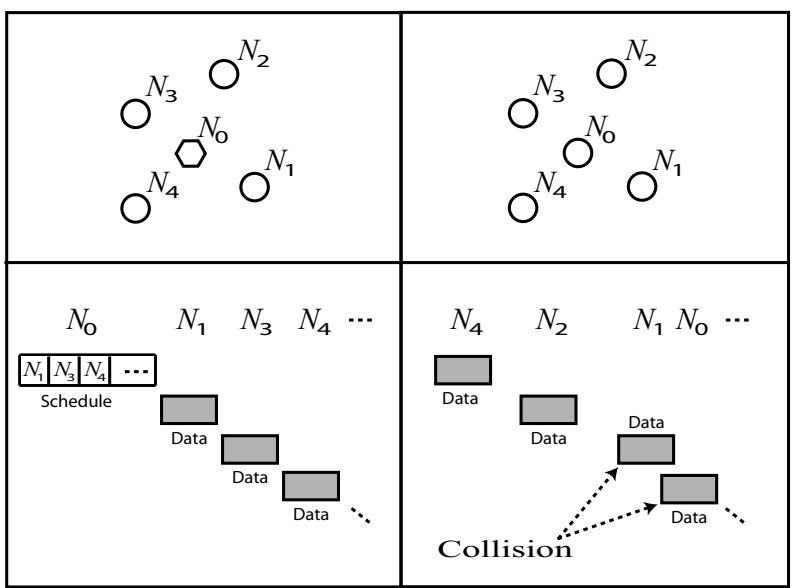

Fig. 1. Illustration of coordinated and non-coordinated MAC protocols. The upper left and right panels show the node distributions for nodes $\mathrm{N}_{0}-\mathrm{N}_{4}$. The lower left panel shows the medium access for the coordinated scheme, where node $\mathrm{N}_{0}$ is the coordinator and the channel access is regulated through a schedule transmitted by $\mathrm{N}_{0}$. The lower right panel shows the channel access for the non-coordinated scheme (e.g., CSMA). Overlapping data transmissions of $\mathrm{N}_{1}$ and $\mathrm{N}_{0}$ lead to a collision. 
Both coordinated and non-coordinated MAC protocols have their advantages and disadvantages.

(i) Collisions are mostly eliminated in coordinated MAC protocols, while frequent packet collisions are unavoidable in non-coordinated protocols, especially under heavy network conditions, which may draw the network into instability in extreme conditions [8].

(ii) The average packet delay using non-coordinated MAC protocols is lower than the average packet delay using coordinated MAC protocols under mild traffic loads. However, under heavy traffic loads, packet delay in noncoordinated protocols rises to very high levels [9].

(iii) The average energy dissipation of nodes in coordinated schemes is significantly lower than in noncoordinated schemes [10].

(iv) Coordinated MAC protocols are more vulnerable to packet losses than non-coordinated MAC protocols due to their dependence on the reliable exchange of control packets, such as the schedule packet. Mobility, multi-path propagation, and channel noise are the main sources of errors that cause packet losses [11].

Although the impact of channel errors on the control packets is crucial to the overall performance of coordinated MAC protocols, evaluation of coordinated MAC protocols under realistic channel errors has found little attention in the literature. In this paper we investigate the effects of channel errors on the control traffic in a coordinated MAC protocol and determine the extent of performance deterioration. Furthermore, we present a comparative performance evaluation of a coordinated and a non-coordinated MAC protocol under a realistic and complete error model.

The general trend in the evaluation of network protocols is to ignore channel errors and assume a perfect channel [12]. Although the assumption of a perfect channel is reasonable in the initial design stage, further verification of a proposed protocol should be conducted with a realistic error model [13]. In this paper we investigate the performance of two MAC protocols, IEEE 802.11 and MH-TRACE (Multi Hop Time Reservation using Adaptive Control for Energy Efficiency), using a realistic error model. IEEE 802.11 is a well-known example of a non-coordinated MAC protocol when it is used for broadcasting. MH-TRACE is a recent example of a coordinated MAC protocol that relies on control packet exchanges for its operation. A comparative evaluation of IEEE 802.11 and MH-TRACE for real-time data broadcasting using a perfect channel showed that the performance of MH-TRACE is better than IEEE 802.11 in terms of throughput and energy efficiency under various network conditions [14]. However, due to the relatively complicated design of MH-TRACE, which relies on robust control packet exchange, the advantages of MH-TRACE over IEEE 802.11 are questionable under a realistic channel error model.

Modeling channel errors in wireless communications has been extensively studied in the literature [15]-[23]. Modeling the channel error as a Markov Process (MP) is a popular and realistic approach in wireless network simulations and has been verified by experimental data. Therefore, we employ a first order Markov Process with two states to evaluate the performance of IEEE 802.11 and MH-TRACE. In this study we want to evaluate the performance of the MAC protocols; thus, the scenario we employ is single hop data broadcasting, which does not require a routing protocol on top of the MAC protocol. Furthermore, in single-hop broadcasting the overall performance (e.g., QoS, energy dissipation) is directly determined by the performance of the MAC protocol. The remainder of this paper is organized as follows. Section II presents the IEEE 802.11 and MH-TRACE MAC protocols. In Section III we investigate the impact of control packet losses by using a rather simplistic error model. We present the complete error model in Section IV. Simulation results and analysis with the complete error model are presented in Section V. Conclusions are drawn in Section VI.

\section{BACKGROUND}

In this section we present an overview of IEEE 802.11 and MH-TRACE when used for single-hop data broadcasting.

\section{A. IEEE 802.11}

In broadcasting mode, IEEE 802.11 uses $p$-persistent CSMA with a constant defer window length (i.e., the default minimum defer period) [4]. When a node has a packet to broadcast, it picks a random defer time and starts to sense the channel. When the channel is sensed idle, the defer timer counts down from the initially selected defer time at the end of each time slot. When the channel is sensed busy, the defer timer is not decremented. Upon the expiration of the defer timer, the packet is broadcast.

\section{B. MH-TRACE}

Multi-Hop Time Reservation Using Adaptive Control for Energy Efficiency (MH-TRACE) is a MAC protocol designed for energy-efficient real-time data broadcasting [14]. Figure 2 shows a snapshot of MH-TRACE clustering and medium access. In MH-TRACE, the network is partitioned into overlapping clusters through a distributed algorithm. Time is organized into cyclic constant duration superframes consisting of several frames. Each clusterhead chooses the least noisy frame to operate within and dynamically changes its frame according to the interference level of the dynamic network. Nodes gain channel access through a dynamically updated and monitored transmission schedule created by the clusterheads, which eliminates packet collisions within the cluster. Collisions with the members of other clusters are also minimized by the clusterheads' selection of the minimal interference frame. Ordinary nodes are not static members of clusters, but they choose the cluster they want to join based on the spatial and temporal characteristics of the traffic, taking into account the proximity of the clusterheads and the availability of the data slots within the corresponding cluster.

Each frame consists of a control sub-frame for transmission of control packets and a contention-free data sub-frame for data transmission (see Figure 3). Beacon packets are used for the announcement of the start of a new frame; Clusterhead Announcement (CA) packets are used for reducing 


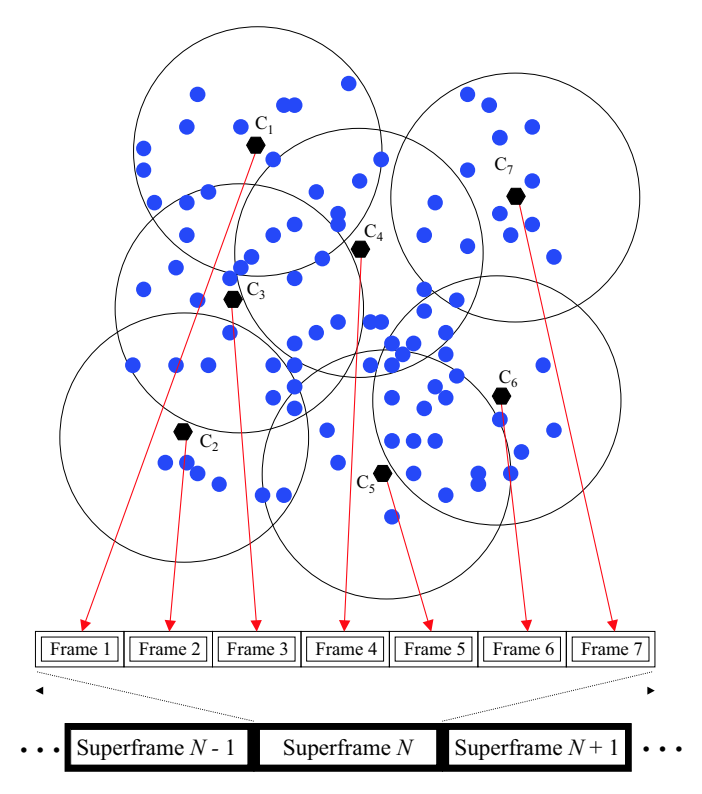

Fig. 2. A snapshot of MH-TRACE clustering and medium access for a portion of an actual distribution of mobile nodes. Nodes $\mathrm{C}_{1}-\mathrm{C}_{7}$ are clusterhead nodes.

co-frame cluster interference; contention slots are used for initial channel access requests; the header packet is used for announcing the data transmission schedule for the current frame; and Information Summarization (IS) packets are used for announcing the upcoming data packets. IS packets are crucial in energy saving. Each scheduled node transmits its data at the reserved data slot.

In MH-TRACE, nodes switch to sleep mode whenever they are not involved in data transmission or reception, which saves the energy that would be wasted in idle mode or in carrier sensing. Ordinary nodes are in the active mode only during the beacon, header, and IS slots. Furthermore, they stay active for the data slots for which they are scheduled to transmit or receive. In addition to these slots, clusterheads stay in the active mode during the CA and contention slots. Instead of frequency division or code division, MH-TRACE clusters use the same spreading code or frequency, and intercluster interference is avoided by using time division among the clusters to enable each node in the network to receive all the desired data packets in its receive range, not just those from nodes in the same cluster. Thus, MH-TRACE clustering does not create hard clusters-the clusters themselves are only used for assigning time slots for nodes to transmit their data.

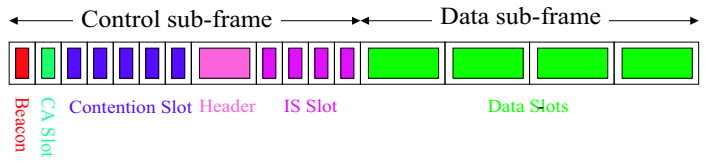

Fig. 3. MH-TRACE frame structure.
TABLE I

SIMULATION PARAMETERS

\begin{tabular}{|l|l|l|}
\hline Acronym & Description & Value \\
\hline$T_{S F}$ & Superframe duration & $25.172 \mathrm{~ms}$ \\
$T_{F}$ & Frame duration & $3.596 \mathrm{~ms}$ \\
$N_{F}$ & Number of frames & 7 \\
$N_{D S}$ & Number of data slots per frame & 7 \\
$N_{C}$ & Number of contention slots per frame & 6 \\
$T_{B}$ & Beacon slot duration & $32 \mathrm{~s}$ \\
$T_{C A}$ & CA slot duration & $32 \mathrm{~s}$ \\
$T_{C}$ & Contention sub-slot duration & $32 \mathrm{~s}$ \\
$T_{H}$ & Header slot duration & $92 \mathrm{~s}$ \\
$T_{I S}$ & IS sub-slot duration & $32 \mathrm{~s}$ \\
$T_{D}$ & Data slot duration & $432 \mathrm{~s}$ \\
$I F S$ & Inter-frame space & $16 \mathrm{~s}$ \\
$T_{d r o p}$ & Packet drop threshold & $50 \mathrm{~ms}$ \\
$N_{F}$ & Number of frames within superframe & 7 \\
$T_{V F}$ & Voice packet generation period & $25.172 \mathrm{~ms}$ \\
$P_{T}$ & Transmit power & $0.6 \mathrm{~W}$ \\
$P_{R}$ & Receive power & $0.3 \mathrm{~W}$ \\
$P_{I}$ & Idle power & $0.1 \mathrm{~W}$ \\
$P_{S}$ & Sleep power & $0.0 \mathrm{~W}$ \\
$D_{T r}$ & Transmission range & $250 \mathrm{~m}$ \\
$D_{C S}$ & Carrier Sense range & $507 \mathrm{~m}$ \\
\hline
\end{tabular}

\section{Effects of Losing Control Packets}

In this section we investigate the effects of control packet losses on protocol performance. Since the non-coordinated MAC protocol (IEEE 802.11) does not utilize control packets, this section focuses on the coordinated MAC protocol (MHTRACE), exclusively. We consider a real-time voice broadcasting application, where each voice packet has a delay constraint and must be dropped after the transmission delay exceeds a certain threshold.

MH-TRACE control packets have vital importance in keeping the clustering and the scheduling mechanisms intact. However, it is not obvious what types of effects the different control packets have on protocol performance. Average network throughput, which is the total number of data packets received by all the nodes in the network, is used as the performance metric. This metric is appropriate for a single hop broadcasting scheme because errors in the control packets will directly affect the number of transmitted data packets since nodes may not be assigned channel access in a timely manner and will thus need to drop packets. As a result of dropped data packets, the number of received data packets will drop linearly. Therefore, the simulations in this section provide a better understanding of the vulnerability of the system to control packet losses.

For the simulations in this section we use a six node fully connected static network to clearly observe the effects of packet losses. When there are no channel errors, all nodes should be able to transmit and receive without any packet drops or collisions. There will be only one clusterhead in the network due to the fact that there cannot be two clusterheads that can hear each other directly. We used the $n s-2$ simulator to evaluate the system performance. Simulation parameters are given in Table I. The channel rate is set to $2 \mathrm{Mbps}$, and we simulated conversational voice coded at $32 \mathrm{Kbps}$, which corresponds to one voice packet per superframe. The simulations are run for $1000 \mathrm{~s}$ and repeated with the same parameters five times. 
Beacon, header and contention packets form the backbone of the protocol and enable MH-TRACE to operate efficiently. Thus, we investigate the effects of losing each of these control packets on the performance of MH-TRACE.

\section{A. Beacon}

Beacon packets are used to announce the existence and continuation of the clusterheads to the nodes in the transmit range of the clusterheads. Since the beacon packet is the main control packet for clusterheads to inform the other nodes about their existence, the stability of a cluster depends on the successful transmission and reception of beacon packets. When an ordinary node cannot receive beacon packets from any of the clusterheads in its receive range, it continues to operate normally for the next superframe until it fails to receive a beacon packet for a second time, sequentially. At the beginning of the next superframe after missing two beacon packets, the node goes into the startup state that will lead to the formation of a new cluster. At this point the node picks a random time to transmit its own beacon packet to contend to become a clusterhead, and it begins to listen to the channel. If another node's beacon is heard in this period, then the node just stops its timer and starts normal operation. Otherwise, when the timer expires, the node sends a beacon and assumes the clusterhead position. Furthermore, when two clusterheads enter in each other's receive range, the one that receives the other's beacon first resigns. These mechanisms ensure the continuity of the protocol in the face of clusterhead failure due to node failure or the clusterhead moving out of range of other nodes in the cluster. However, the probability for a node to miss three consecutive beacons due to channel errors and overtake the clusterhead position when the clusterhead is actually still available is very low.

Other than clusterhead stability, missing beacon packets also slightly increases the average packet delay when two successive beacons are missed. Increase in delay is mainly due to the fact that the node loses time when it enters into startup mode. On the other hand, missing the beacon once does not affect the node at all, provided that the header is successfully received. Since the header contains all the required information about the data transmission schedule, losing beacon packets actually has very little effect on throughput.

We ran simulations using uniform packet error probabilities for beacon reception (i.e., only beacon packets experience channel errors and all the other packets are error free). Although this error model is rather simplistic, it is sufficient for our purposes in this section. We utilized $1.0 \%, 3.0 \%$, and $5.0 \%$ packet error probabilities. Note that a $5.0 \%$ packet error probability represents a harsh environment [11]. However, the simulation results reveal that the performance of MH-TRACE does not deteriorate significantly. Indeed, losing 10,000 beacon packets resulted in only 15 dropped data packets. In Figure 4 the effects of losing beacon packets are presented in normalized form. For example, for each dropped beacon packet, there are only 0.0015 dropped data packets, on average. Note that the data packet drop rate is marked as 0.00 in Figure 4 for beacon packet error since the actual value, 0.0015 , is so small.

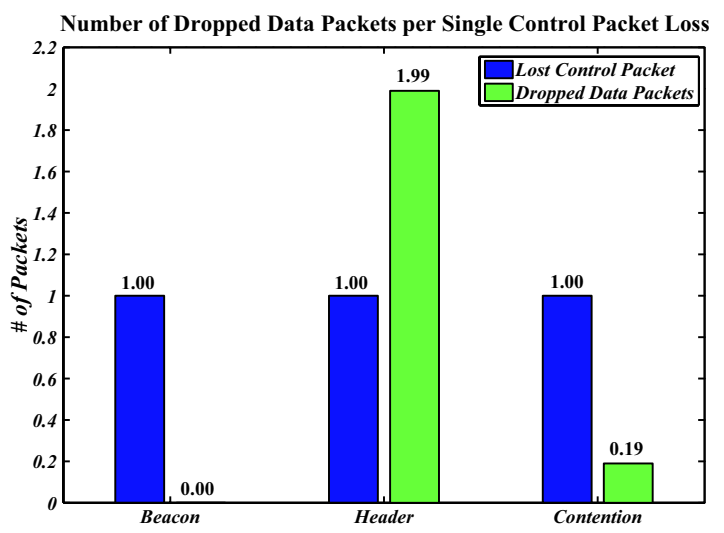

Fig. 4. MH-TRACE performance degradation in terms of dropped data packets for beacon, header, and contention packet losses.

\section{B. Header}

Header packets are sent by the clusterheads to announce the data transmission schedule of the current frame. The transmission schedule is a list of nodes that have data slots reserved in the current frame, along with their data slot numbers. Therefore, missing a header packet puts a node on hold if it has data to send. Since in voice communications delay bounds are stringent, if the waiting time for a voice packet exceeds a certain threshold it is dropped (i.e., $50.0 \mathrm{~ms}$ in this study).

In order to support QoS, once a node obtains a slot its reservation is renewed automatically by the clusterhead as long as the node continues to transmit data. However, when a particular node misses a header packet, it cannot transmit during its reserved data slot and its reservation is cancelled by the clusterhead. Furthermore, a node that misses its reserved data slot still thinks that it has a reserved data slot. Thus, during the next frame when the node does not hear its ID in the schedule, it understands that it needs to contend again in the following superframe. This chain reaction increases the packet delay as much as three superframe times. Since time spent to get a data slot results in increased delay, data packets are dropped when they exceed the packet drop threshold, which is approximately twice the superframe time. Therefore, missing a single header results in at least one, and most probably two, dropped data packets.

In Figure 4 the simulation results obtained with the header packet error model are presented. As expected, the average number of dropped data packets per missed header packet is close to two.

\section{Contention}

Each node contends for channel access when it has data to send but did not reserve a data slot in the previous cyclic superframe. A node randomly chooses a sub-slot to transmit its request. If the contention is successful (i.e., no collisions or error occurred), the clusterhead grants a data slot to the contending node if there are available data slots. If the node ID is not in the schedule, which is embedded in the header packet, the node understands that its contention was unsuccessful and waits for the next contention period. 
We utilized CBR traffic for the evaluation of the impact of channel errors on the beacon and header packets. However, we use a statistical model of a voice activity detection based voice codec to evaluate the impact of channel errors on the contention packets due to the fact that the contention packets are not like beacon or header packets (i.e., contention packets are event triggered rather than periodic). Once a node gets channel access, it will not loose it and will not be using contention packets for the rest of the simulation time. Thus, to prevent this situation, which does not let us monitor the effects of packet losses on bursty traffic, we employed a statistical voice source model. According to the voice source model, speech is classified into "spurts" and "gaps" (i.e., gaps are the silent moments during a conversation). During gaps, no data packets are generated, and during spurts, data packets are generated at $32 \mathrm{Kbps}$ data rate. Both spurts and gaps are exponentially distributed statistically independent random variables, with means $1.0 \mathrm{~s}$ and $1.35 \mathrm{~s}$, respectively [5].

Losing contention packets introduces additional delays into the network and causes data packets with critical delay values to be dropped. However the impact in this case is not as large as for errors in the header packets. Depending on traffic load and node density of the network, the results provided in Figure 4 are subject to change. As the traffic load and node density increase, average delay in the system also increases. Therefore, delay caused by losing contention packets results in a higher probability of dropped data packet.

The next section addresses the design of a realistic wireless channel model, which we will be employing in our detailed simulations.

\section{A REALISTIC WIRELESS CHANNEL MODEL}

In this study we model the wireless channel as a first order Markov process with two states [24]. This model has been frequently employed in the literature to model fading errors at higher layers to calculate the average block error rate (packet error rate) [15]-[23]. In the literature, most of the channel error models are based on the assumption that data packet transmissions are i.i.d. In addition, many coding schemes and protocols were initially designed for i.i.d. channels. It has been shown that the special structure of Markov approximation makes it naturally useful and tractable for this purpose.

The Markov chain assumes that an adequate description of a system is given by a finite number of states. Each state is assigned a probability of the system being in that state. For example, the typical movement of the stock market could be considered as a simple two-state model in terms of up and down movement of the index. The study of Markov approximation for fading channels dates back to the early work of Gilbert [15] and Elliott [16], who built a two-state Markov channel known as the Gilbert-Elliott channel. In a simplified Gilbert model [17], the error probabilities in "bad" and "good" states are 1 and 0 , respectively. Assuming 1 and 0 denote successful and erroneous transmission in a given slot, the state transition diagram is shown in Figure 5. The Normal state represents a perfect channel in which there is no error present, whereas the Lossy state represents a wireless channel

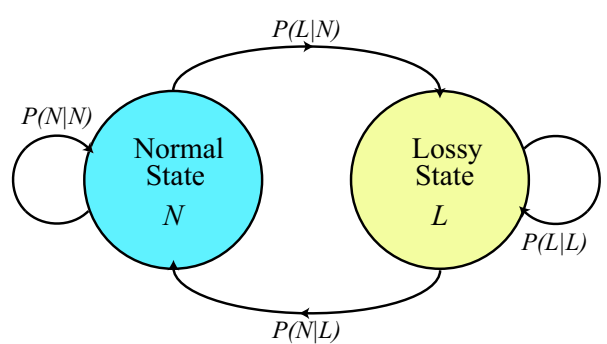

Fig. 5. Gilbert-Elliot channel model.

in which no packet can be delivered without error. The channel statistics are controlled by a set of transition probabilities that determine the individual probabilities $P(N)$ and $P(L)$.

The entropy rate of discrete finite random variables $\left(X_{1}, X_{2}, \ldots, X_{N}\right)$ is defined as:

$$
H_{0}=\lim _{N \rightarrow \infty} \frac{H\left(X_{1}, X_{2}, \ldots, X_{N}\right)}{N} .
$$

If the random variables are stationary, we have

$$
H_{0}=\lim _{N \rightarrow \infty} H\left(X_{N} \mid X_{1}, \ldots, X_{N-1}\right),
$$

and in the case of stationary Markov sequence we have

$$
H_{0}=\lim _{N \rightarrow \infty} H\left(X_{N} \mid X_{N-1}\right)=H\left(X_{2} \mid X_{1}\right) .
$$

For a stationary Markov sequence [24], the net probability flow between the two states is zero once the stationary distribution has been reached (i.e. Entropy of a state is constant at equilibrium). In our case we have four equations (Equations (4)-(7)) with six unknowns, and therefore, it is possible to assign the desired stationary probabilities to both states $\{P(N), P(L)\}$ and calculate the transition probabilities $\{P(N \mid L), P(L \mid N)\}$ accordingly.

$$
\begin{gathered}
P(N) \cdot P(L \mid N)=P(L) \cdot P(N \mid L), \\
P(N)+P(L)=1, \\
P(N \mid N)+P(N \mid L)=1, \\
P(L \mid L)+P(L \mid N)=1 .
\end{gathered}
$$

Using the two state model, one can generate a simulated wireless channel behavior for the protocol under study and perform realistic simulations. In Figure 6, the state transition behavior of the Gilbert model is illustrated. The channel

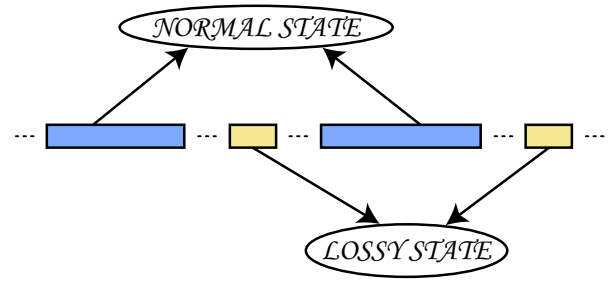

Fig. 6. State transition with the Gilbert-Elliot channel model. 
spends some percentage of the total simulation time, determined by $P(N)$, in the normal state $(N)$ and some time determined by $P(L)$ in the lossy state $(L)$. Moreover, errors occur in bursts due to the fact that the channel spends portions of time in both states. When the channel is in the lossy state, errors are introduced according to the length of the packet: the probability of error for a longer packet is higher than the probability of error for a shorter packet [25], [26]. In other words, it is more likely for a data packet to be in error than for a beacon packet, which is the shortest packet in MH-TRACE, to be in error.

For the rest of the simulations Gilbert model is employed with transition probabilities and state probability distribution shown in Table II. The state probabilities $P(N)$ and $P(L)$ can be set according to the requirements by using Equations (4)(7).

\section{EVALUATION OF IEEE 802.11 AND MH-TRACE}

In this section we present the simulation results and analysis of IEEE 802.11 and MH-TRACE for a $1 \mathrm{~km}$ by $1 \mathrm{~km}$ area network with 80-200 nodes using the parameters in Table I. In the first set of simulations, we evaluate the protocols' performance using a static network, while in the second set of simulations, we use a Random Way-Point mobility model with node speeds chosen from a uniform distribution between 0.0 $\mathrm{m} / \mathrm{s}$ and $5.0 \mathrm{~m} / \mathrm{s}$ (the average pace of a marathon runner) with zero pause time. We used the statistical voice source model described in Section III-C. The simulations are repeated with the same parameters five times, and the data points in the figures are the average of the ensemble and the error bars are the standard deviation of the ensemble. Table III presents the key simulation settings for the two sets of simulations.

Beacon, CA, contention, and IS packets are all 4 bytes. The header packet has a variable length of 4-18 bytes, consisting of 4 bytes of packet header and 2 bytes of data for each node to be scheduled. Data packets are 104 bytes long, consisting of 4 bytes of packet header and 100 bytes of data. Each slot or sub-slot includes $16 \mathrm{sec}$ of guard band (IFS) to account for switching and round-trip time.

\section{A. SET1: Network with Stationary Nodes}

In this section the throughput of the two protocols (IEEE 802.11 and MH-TRACE) are compared for a network of stationary nodes. The protocols are simulated using both a perfect and a lossy channel whose statistics are given in Table II (i.e., the channel spends $6.25 \%$ of the time in the lossy state and rest of the time, $93.75 \%$, the channel is in the normal state). The parameters of the channel model are taken from the empirical data presented in [21]. The ratio of the time spent in the lossy and normal states affects the severity of the

TABLE II

GILBERT-ELLIOT CHANNEL MODEL STATISTICS

\begin{tabular}{c|c|c|c}
\hline State & $\mathbf{P}($ State $)$ & $\mathbf{P}(\boldsymbol{L} \mid$ State $)$ & $\mathbf{P}(\boldsymbol{N} \mid$ State $)$ \\
\hline $\boldsymbol{N}$ (Normal) & 0.9375 & 0.01 & 0.99 \\
\hline $\boldsymbol{L}$ (Lossy) & 0.0625 & 0.85 & 0.15 \\
\hline
\end{tabular}

TABLE III

SimULATION SETUP

\begin{tabular}{ccc}
\hline PARAMETER & SET 1 & SET 2 \\
\hline Protocol & MH-TRACE/ & MH-TRACE/ \\
& IEEE 802.11 & IEEE 802.11 \\
Number of Nodes & $80-200$ & $80-200$ \\
Simulation Time & $200 \mathrm{~s}$ & $200 \mathrm{~s}$ \\
Number of Repetition & 5 & 5 \\
Error Model & None/Gilbert & None/Gilbert \\
Node Mobility & Stationary & Mobile \\
\hline
\end{tabular}

channel. For example, in a two node scenario, where one node continuously transmits data packets and the other listens, the packet loss ratio is directly determined by the percentage of time spent in the lossy state (e.g., $6.25 \%$ of the packets are lost with a channel that spends $6.25 \%$ of the time in the lossy mode, given that the probability of data packet loss in the lossy state is close to unity). Moreover, the probability that a packet is in error also depends on the length of the packet (i.e., short packets such as beacon and contention packets have smaller probabilities of being in error than longer packets such as data packets). In the lossy state, the probability of dropping a data packet is unity, the probability of dropping a header packet is 0.18 , and the rest of the control packets have a 0.04 probability of error.

Figure 7 presents the average number of received packets per node per second versus the number of nodes. The curves labeled as "no error" are associated with the perfect channel scenario, whereas the ones labeled as "error" are obtained using the lossy channel model. When there are only 80 nodes with a perfect channel, the throughput of MH-TRACE (197.26 \pm 3.26 packets/node/s) is $9.16 \%$ more than that of 802.11 (180.71 \pm 0.01 packets/node/s). As the number of nodes increases, the difference between the two protocols increases. For 200 nodes MH-TRACE outperforms 802.11 by a factor of 2.2 in terms of received packets per node per second. IEEE 802.11 throughput is lower than MH-TRACE throughput due to excessive collisions experienced by IEEE 802.11.

For the lossy channel, the throughput of both protocols deteriorates in comparison to the performance with a lossless

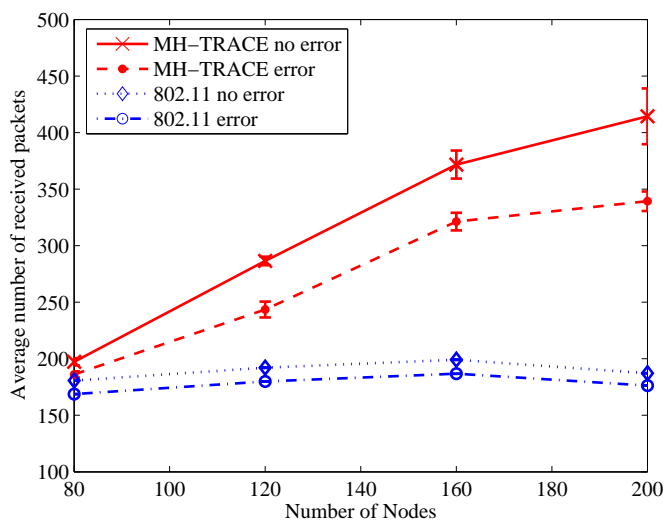

Fig. 7. SET 1 (stationary nodes): Average number of received packets per node per second versus number of nodes. 


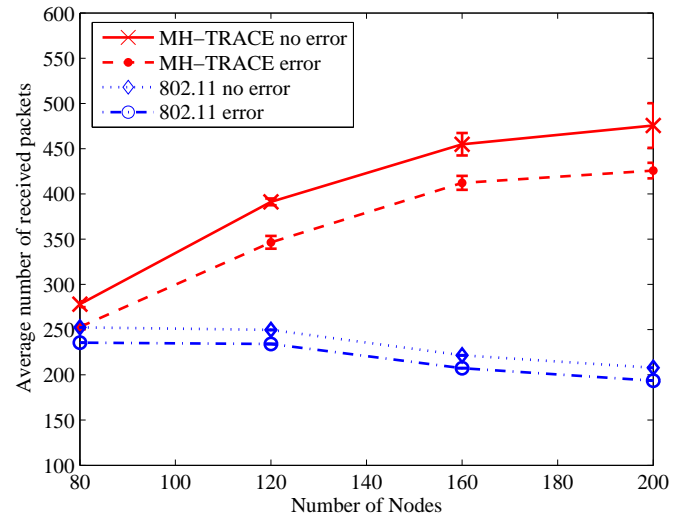

Fig. 8. SET 2 (mobile nodes): Average number of received packets per node per second versus number of nodes.

channel, but the loss in MH-TRACE is larger than the loss in 802.11 . When the number of nodes is 80 , both protocols loose approximately $6.3 \%$ of their performance under perfect channel conditions. Note that the channel stays in the lossy state approximately $6.3 \%$ of the total time, and all the data packets are dropped during the lossy state. On the other hand, control packets are dropped with less probability since packet loss probability is directly related with the packet length. With an increase in the number of nodes, both control and data traffic of MH-TRACE become heavier and the number of dropped packets increase. Therefore, channel errors degrade the performance by nearly $18.1 \%$, whereas throughput loss in IEEE 802.11 remains at the same level (6.3 \%). Thus, approximately $12 \%$ of the packet losses in MH-TRACE are due to the loss of control packets. The dominant factor is the header packet losses. Despite the fact that MH-TRACE throughput is reduced from 414 to 339 packets/node/s, it still performs $92.5 \%$ better than IEEE 802.11.

\section{B. SET2: Network with Mobile Nodes}

In this set of simulations we evaluate the protocols' performance in terms of throughput, stability, data packet delay, and energy dissipation for a network of mobile nodes.

Figure 8 shows the throughput of IEEE 802.11 and $\mathrm{MH}-$ TRACE as a function of the number of nodes in the network. Due to node mobility, the throughput of both protocols is reduced slightly, both in the lossless and lossy channel conditions. However, the general trend seen in the static case is preserved.

Figure 9 and Figure 10 show the average clusterhead lifetime and the average number of clusterheads over all simulation time, respectively. These metrics are related to the stability of the clustering algorithm of MH-TRACE. Although there are small differences between the lossless and lossy cases in the behavior of the clustering structure, these differences are not significant. Thus, channel errors do not affect the clustering algorithm of MH-TRACE significantly; rather the channel access mechanism is most affected from the channel errors, which manifests itself by a slight reduction in the throughput in excess of the IEEE 802.11 throughput reduction. The discussion on the effects of control packet losses in

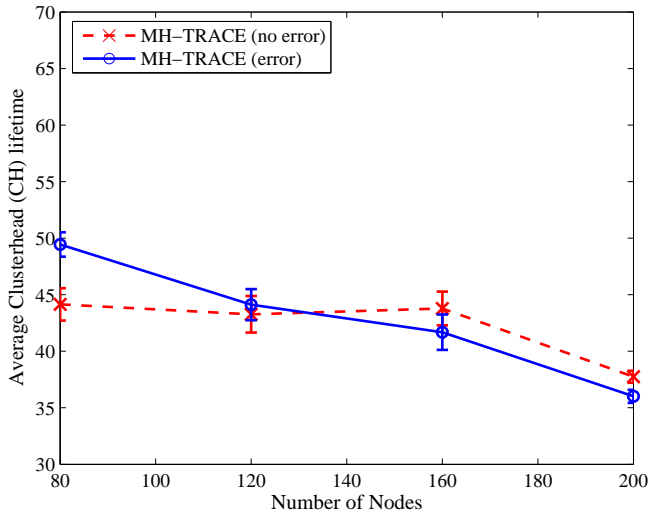

Fig. 9. SET 2 (mobile nodes): Average life time of clusterheads versus number of nodes.

Section III, in particular, the impact of beacon and header packet losses, supports this argument. Header packet losses, which are related with channel access, cause more data packet losses than beacon packet losses, which are related with the clustering mechanism.

One of the most important advantages of MH-TRACE over IEEE 802.11 is its better energy efficiency. The average energy dissipation of MH-TRACE and IEEE 802.11 with lossless and lossy channel conditions as a function of node density is presented in Figure 11. Energy dissipations of both protocols are insensitive to the channel conditions in the application scenario we considered. Nevertheless, MH-TRACE energy dissipation stays less than $40 \%$ of the energy dissipation of IEEE 802.11 energy dissipation.

Figure 12 shows the average data packet delay for $\mathrm{MH}-$ TRACE and IEEE 802.11 as a function of node density. Note that the maximum packet delay is $50 \mathrm{~ms}$, which is dictated by the application layer. MH-TRACE packet delay is higher than IEEE 802.11 packet delay in both lossless and lossy channel conditions due to the fact that in MH-TRACE nodes can have channel access only once in a superframe time, whereas in IEEE 802.11 channel access is not restricted. Both MHTRACE and IEEE 802.11 have comparatively higher packet delays in a lossy channel. The increase in the packet delay

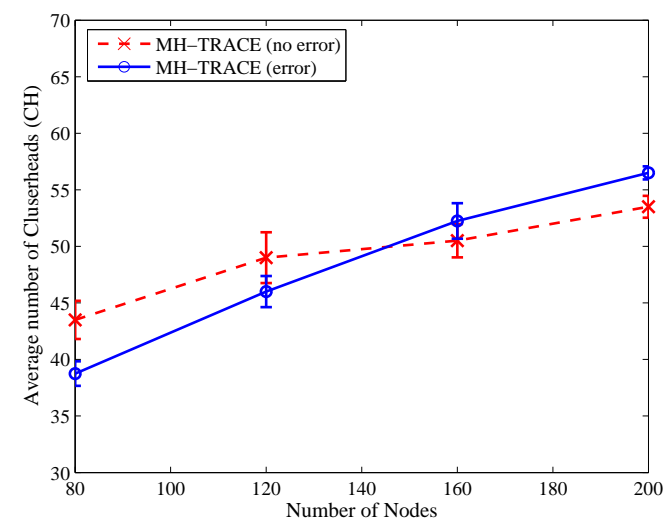

Fig. 10. SET 2 (mobile nodes): Average number of clusterheads versus number of nodes. 


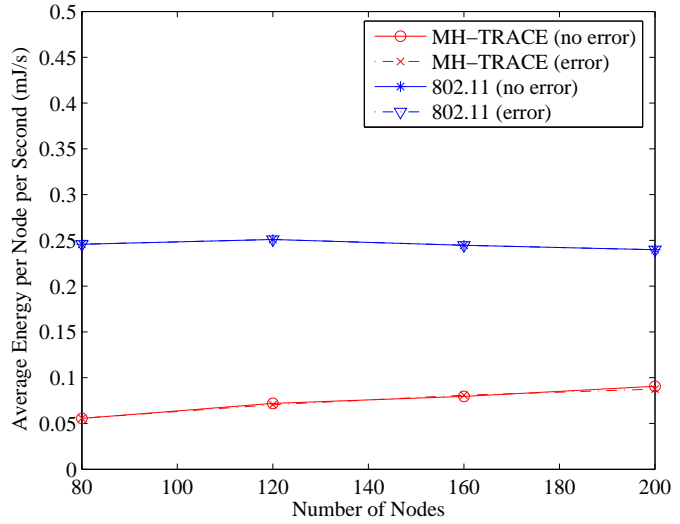

Fig. 11. SET 2 (mobile nodes): Average energy consumption per node per second versus number of nodes.

in MH-TRACE is mainly due to the header packet losses, as once a node looses a header packet, it loses several frame times before regaining channel access.

\section{CONCLUSION}

In this paper we investigated the impact of channel errors on the performance of MH-TRACE and IEEE 802.11, which are examples of coordinated and non-coordinated MAC protocols, respectively, through $n s-2$ simulations using the Gilbert-Elliot channel model. As expected, the impact of channel errors is more severe on MH-TRACE than IEEE 802.11 due to the dependence of MH-TRACE on robust control packet traffic. Nevertheless, the performance of MH-TRACE remains superior to that of IEEE 802.11, even in the presence of large channel errors. Hence, the major conclusion of this study is that coordinated MAC protocols are preferable over non-coordinated MAC protocols even under noisy channel conditions. If the channel conditions improve due to either a better channel or forward error correction utilized in the control traffic, then MH-TRACE performance loss due to the non-perfect channel will be similar to that of IEEE 802.11. Our future work will focus on the evaluation of networkwide broadcast routing algorithms using coordinated and noncoordinated MAC protocols under noisy channel conditions.

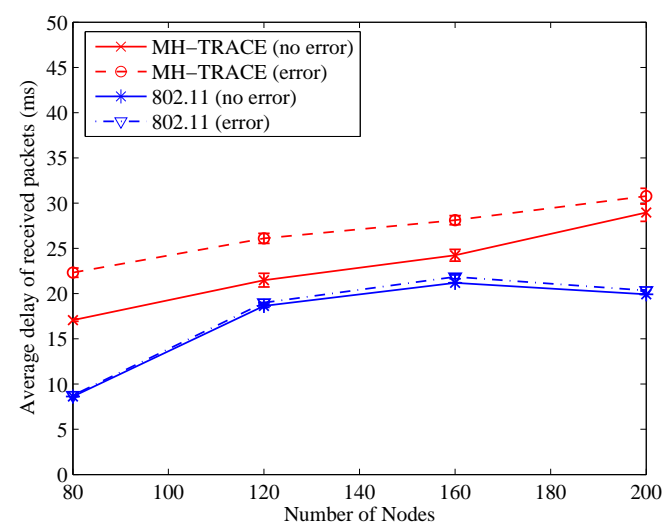

Fig. 12. SET 2 (mobile nodes): Average data packet delay versus number of nodes.

\section{REFERENCES}

[1] H. Zimmermann. Osi reference model - the iso model of architecture for open systems interconnection. IEEE Transactions on Communications, 28(4):425-432, Apr 1980.

[2] A. Chandra, V. Gummalla, and J. O. Limb. Wireless medium access control protocols. IEEE Communications Surveys and Tutorials, 3:215, 2000.

[3] W. Stallings. Wireless Communications and Networks. Prentice-Hall, 2002.

[4] B. O'Hara and A. Petrick. The IEEE 802.11 Handbook: A Designer's Companion. IEEE Press, 1999.

[5] D. J. Goodman and S. X. Wei. Efficiency of packet reservation multiple access. IEEE Trans. Vehic. Tech., 40(1):170-176, Feb 1991.

[6] C. R. Lin and M. Gerla. Adaptive clustering for mobile wireless networks. IEEE Journal of Selected Areas in Communications, 15(7):1265$1275,1997$.

[7] T. Cooklev. Wireless Communication Standarts. IEEE Press, 2004.

[8] B. Tavli and W. B. Heinzelman. TRACE: Time reservation using adaptive control for energy efficiency. IEEE Journal on Selected Areas of Communications, 21:1506-1515, Dec 2003.

[9] V. Kanodia, C. Li, A. Sabharwal, B. Sadaghi, and E. Knightly. Distributed multi-hop scheduling and medium access with delay and throughput constraints. In Proceedings of the Seventh Annual International Conference on Mobile Computing and Networking (MobiCom), 2001.

[10] J.-C. Chen, K. M. Sivalingam, P. Agrawal, and S. Kishore. A comparison of mac protocols for wireless local networks based on battery power consumption. In Proceedings of the Seventeenth International Annual Joint Conference of the IEEE Computer and Communications Societies (INFOCOM), 1998

[11] D. A. Maltz, J. Broch, and D. B. Johnson. Lessons from a full-scale multihop wireless ad hoc network testbed. IEEE Personal Commun. Mag., 8(1):8-15, Feb 2001.

[12] D. Kotz, C. Newport, and C. Elliot. The mistaken axioms of wirelessnetwork research. Technical Report TR2003-467, Dartmouth College Computer Science Department, Jul 2003.

[13] R. S. Gray, D. Kotz, C. Newport, N. Dubrovsky, A. Fiske, J. Liu, C. Masone, S. McGrath, and Y Yuan. Outdoor experimental comparison of four ad hoc routing algorithms. In Proceedings of the Seventh ACM/IEEE International Symposium on Modeling, Analysis, and Simulation of Wireless and Mobile Systems (MSWiM), 2004.

[14] B. Tavli and W. B. Heinzelman. MH-TRACE: Multi hop time reservation using adaptive control for energy efficiency. IEEE Journal on Selected Areas of Communications, 22(5):942-953, June 2004.

[15] E. N. Gillbert. Capacity of a burst-noise channel. Bell System Tech. J., 39(9):1253-65, Sept 1960.

[16] E. O. Elliott. Estimates of error rates for codes on bursty-noise channels. Bell System Tech. J., 42(9):1977-97, Sept 1963.

[17] J. R. Yee and E. J. Weldon. Evaluation of the performance of error correcting codes on a gilbert channel. IEEE Trans. Commun., 43(8):2316-23, Aug 1995.

[18] J. L. Lemmon. Wireless link statistical bit error model. Technical Report NTIA Report 02-394, U.S. Department of Commerce, Jun 2002.

[19] M. Zorzi and R. R. Rao. Lateness of probability of a retransmission scheme for error control on a two-state markov channel. IEEE Trans. Commun., 47(10):1537-48, Oct 1999.

[20] J. McDougall and S. Miller. Sensitivity of wireless network simulations to a two-state markov model channel approximation. In Proceedings of the Global Telecommunications Conference (GLOBECOM), 2003.

[21] A. Konrad, B. Zhao, A. Joseph, and R. Ludwig. A Markov-based channel model algorithm for wireless networks. Wireless Networks, 9(3):189-199, May 2003.

[22] A. Gurtov and S. Floyd. Modeling wireless links for transport protocols. ACM Computer Communication Review, 34(2):85-96, 2004.

[23] E. Modiano. An adaptive algorithm for optimizing the packet size used in wireless arq protocols. Wireless Networks, 5(4):279-286, 1999.

[24] T. Cover and J. Thomas. Elements of Information Theory. New York: Wiley, 1991.

[25] J. G. Proakis. Digital Communications. New York: Mc Graw-Hill, 1989.

[26] P. Lettieri and M. B. Srivastava. Adaptive frame length control for improving wireless link throughput, range, and energy efficiency. In Proceedings of the Seventeenth International Annual Joint Conference of the IEEE Computer and Communications Societies (INFOCOM), 1998. 